Volume: 1 | Volumen 1 | Número 1 | Number 1 | pp. $47-62$ ISSN: 2634-355X (Print) | ISSN: 2634-3568 (Online) journals.tplondon.com/yeiya

First Submitted: 14 September 2020 Accepted: 29 November 2020 DOI: https://doi.org/10.33182/y.v1i1.1252

\title{
Los efectos de la financiarizacion sobre la naturaleza en el contexto de la pandemia COVID-19
}

\author{
Gabriel Alberto Rosas Sánchez ${ }^{1}$
}

\section{Resumen}

El objetivo de este trabajo es mostrar las repercusiones de la financiarización sobre la velocidad e intensidad de explotación de la naturaleza. Bajo una visión antropocéntrica, los ecosistemas reducidos a su expresión monetaria se vuelven objeto de rentabilidad y especulación. La crisis financiera del 2007-2008, cuyos efectos persisten y se magnificaron a raíz de la crisis sanitaria de la COVID-19, genera las condiciones propicias para acelerar el proceso de financiarización del sistema ecológico al convertirse en vía de rentabilidad de corto plazo. El riesgo de la pandemia radica en la profundización de los mecanismos de financiarización ecológica como salida de la crisis económica y climática a partir de las normas y objetivos operativos que rigen el funcionamiento de los principales acuerdos internacionales en materia ambiental.

Palabras clave: financiarización; naturaleza; COVID-19

\section{Abstract \\ Financialization effects on naturalization in the context of COVID-19}

The objective of this paper is to show the repercussions of financialization on the speed and intensity of exploitation of nature. Under an anthropocentric vision, ecosystems reduced to their monetary expression become the object of profitability and speculation. The financial crisis of 2007-2008, whose effects persist and were magnified as a result of the health crisis of COVID-19, creates the conditions to accelerate the process of financialization of the ecological system by becoming a means of short-term profitability. The risk of the pandemic lies in the deepening of the ecological financialization mechanisms as a way out of the economic and climate crisis based on the rules and operational objectives that govern the operation of the main international agreements on environmental matters.

Keywords: financialization; nature; COVID-19

\section{Introducción: contexto de la financiarización y la pandemia}

El ascenso de las actividades financieras sobre la estructura económica real ha dejado en el camino severos desenlaces durante los últimos años. El transcurso de la década de los años noventa tuvo como protagonistas de la crisis a países emergentes quienes habían enseñado la facilidad que se podía perder la soberanía estatal (Tooze, 2018), convirtiéndose en ejemplo del proceder incorrecto en materia económica.

Las lecciones no se quisieron aprender; por el contrario, la desregulación de las actividades financieras se convirtió en el vehículo conductor de la política y un pilar fundamental de la competitividad económica nacional ${ }^{2}$. El ascenso de las actividades financieras de manera

\footnotetext{
${ }^{1}$ Universidad Autónoma Metropolitana. Ciudad de México, México. Correo electrónico: rosassanchezgabriel@gmail.com

${ }^{2}$ Véase por ejemplo los criterios de competitividad establecidos en cada reporte de competitividad global publicado por el Foro Económico Mundial donde destaca el papel del sistema financiero.
} 
asimétrica respecto a las actividades reales de la economía provocó la consolidación de un árbol con raíces extremadamente frágiles. La extensión y dominio de estas raíces sobre la estructura social, política y principalmente económica (producción de mercancías) se define en este trabajo como financiarización.

Ese proceso desplaza a las actividades del sector real, convirtiéndose este último únicamente en referencia para seguir generando expectativas de ganancias. El caos que puede provocar el sector financiero radica en la especulación sobre el rendimiento futuro, es decir, en el juego donde los inversores intentan predecir el precio actual de un instrumento bursátil considerando la variación de su precio futuro. La brecha entre las expectativas de ganancias y las expectativas de los precios futuros en un elemento que puede desencadenar en burbujas especulativas. La euforia que impacta sobre el comportamiento de los inversores termina por controlar el destino de la economía real a costa de daños sociales inmensos cuando la burbuja estalla, mientras en épocas de bonanza las ganancias se acumulan de manera privada.

La evolución tecnológica del siglo XX creó las condiciones idóneas para la movilidad a gran escala de activos financieros, la creación de sofisticados instrumentos de inversión y, sobre todo, la capacidad de ejecutar acciones coordinadas mundialmente de manera instantánea gracias a la interconexión de los mercados financieros mundiales.

El contexto actual es tan endeble que la movilidad de capitales depende más de las conexiones de internet y no de la regulación gubernamental. El caso reciente y catastrófico en la época actual del capitalismo es la crisis del 2008. El vínculo entre múltiples bancas de inversión y agencias de seguros en colusión de las bancas centrales al proveer dinero barato (Tooze, 2018), convirtieron al sector inmobiliario en objeto de especulación que provocó en el momento de euforia una ola de créditos sin ninguna garantía de pago, mientras que el sector crecía sustentado en cimientos de castillos ficticios. Como resultado, millones de personas perdieron sus trabajos, familias quedaron sin casas, los niveles de ingreso nacional cayeron y siguen sin recuperarse a pesar de los miles de millones de dólares gastados por los gobiernos para evitar que la crisis tomara mayores dimensiones. Las consecuencias de la crisis persisten y la pandemia de la COVID-19 vino a reivindicar y profundizar el hecho que la turbulencia nunca desapareció por completo.

A pesar de los daños por el comportamiento desmedido del sector financiero las economías del mundo siguen considerando los resultados de las bolsas de valores como indicadores adecuados del desempeño de la economía real. Siguiendo a Jeannot (2020) la economía numérica ha tomado auge al considerar los movimientos de capital, tasas de cambio y líneas de crédito el timón de la economía en lugar que sea la economía real productora de bienes y servicios.

El increíble comportamiento de esta economía simbólica pudo verse en meses recientes donde, en plena contingencia acompañada de caída de la producción, empleos e incremento de la población en condiciones de pobreza, los indicadores bursátiles como el Nasdaq celebraron máximos históricos ${ }^{3}$, haciendo creer que la economía iba en plena recuperación. Mientras el Informe de prospectivas globales de crecimiento del Banco Mundial (2020) estima caídas en el Producto Interno Bruto real mundial del 5.2 \%, para la Zona Euro del 6.1\% y

\footnotetext{
${ }^{3}$ De acuerdo con información de Bloomberg, el mes de julio de 2020 el Nasdaq Composite registró un máximo histórico (10,767 puntos) manteniendo semanas al alza, incluso llegando a los 11 mil puntos por primera vez en su historia el mes de agosto, mientras el S\&P 500 inició el mes de septiembre en 3,526 unidades, un nuevo registro histórico.
} 
América Latina del 7.2\%. La discordancia entre el desempeño de ambas economías puede catalogarse como cínica y fuera de lugar.

Frente al panorama sanitario que desafía el mundo, los riesgos de la financiarización son latentes. En los últimos años se han incorporado nuevos elementos en busca de la rentabilidad de corto plazo, entre ellos, la naturaleza en sus múltiples expresiones (Bruckmann, 2016). Desde el inicio del siglo un espacio preferido en los portafolios de inversión ha sido el sector agrícola, en especial, los denominados commodities. El mercado de futuros del maíz, trigo, arroz, soya, entre otros granos han venido en ascenso, comprometiendo las cosechas de muchos años por delante y generando sobreexplotación de las tierras para mantener el nivel de productividad. Es decir, el pacto presente de cierta cantidad de productos naturales en las bolsas de valores obliga a los productores a mantener o incluso incrementar los niveles de rendimiento a través de la intensificación de los procesos productivos y con ello comprometiendo el equilibrio ecológico futuro a costa del mayor deterioro medioambiental.

Este trabajo intenta mostrar el riesgo de la financiarización sobre el medio ambiente en contexto de crisis como el que atraviesa el mundo magnificada por la presencia del SARSCoV-2. El argumento principal del presente refiere al posible riesgo que conlleva la obsesión por el desempeño de la economía simbólica sobre la economía real provocando que el sector financiero sea la vía de corto plazo para la recuperación económica mundial sustentado en el rendimiento de los principales indicadores bursátiles sobre expectativas de ganancias futuras. En este proceso, el medio ambiente ve incrementado su vulnerabilidad al ser tratado como una mercancía sujeta a la valorización por su potencial de rentabilidad en el corto plazo y de menor incertidumbre gracias a su reducción monetaria en los mercados internacionales dejando de lado su importancia cultural y esencial para la vida misma del ser humano y las especies naturales.

Esta presión puede generar un proceso intensivo y extractivo sobre los ecosistemas por considerarse vía de emergencia frente al contexto de la pandemia. El riesgo recae sobre el papel de la especulación sobre los precios de los bienes naturales, así como la posibilidad de crear un nuevo episodio de especulación como aconteció en la crisis del 2008 sobre los precios de los commodities que afectó severamente a países latinoamericanos. Los principales acuerdos en materia ambiental promueven dentro de sus principios de acción procesos que intensifican la financiarización. Para justificar esta idea el trabajo se desarrolla en 6 apartados. El primero refiere al rol de la desregulación de las actividades financieras, el segundo apartado se aborda de manera detallada el proceso y mecanismos de la financiarización. En tercer lugar, se presentan los riesgos e implicaciones de este fenómeno sobre la economía, sociedad y medio ambiente. El cuarto inciso se profundiza en los riesgos de la financiarización sobre la naturaleza y las posibles consecuencias sobre el agravamiento del deterioro ambiental. El quinto apartado consiste en una reflexión de la financiarización del medio ambiente en el contexto de la pandemia sanitaria actual como una herramienta de recuperación económica y el peligro que implica para los ecosistemas en beneficio de los rentistas financieros. Por último, el texto concluye con reflexiones finales.

\section{La desregulación financiera}

Durante los últimos años el mundo ha sufrido las consecuencias de la euforia financiera. Cabe preguntarse ¿Qué hay detrás de ello? De acuerdo con Kindleberger y Aliber (2005) las crisis financieras y quiebras bancarias de los últimos treinta años fueron de carácter sistemático a 


\section{Los efectos de la financiarizacion sobre la naturaleza en el contexto de la pandemia COVID-19}

causa principalmente a la implosión de las burbujas de precios de los activos y especulación en los mercados bancarios. El origen del gran desconcierto internacional puede asociarse particularmente a la desregulación financiera.

La Gran Depresión en Estados Unidos durante los años treinta trajo serias lecciones sobre el daño que puede causar el libre actuar de las finanzas. Frente a las corridas bancarias previas al colapso de 1929 la regulación se convirtió en aspecto relevante. En 1933 surge la Ley GlassSteagall que puso límites a la tasa de interés de los créditos y ofreció garantías a los depósitos de los consumidores. Al año siguiente se consolidó una serie de reformas complementarias como fueron la Securities Exchange Act y la Securities and Exchange Commission que regularon las actividades secundarias de la bolsa de valores. En la misma década se establecieron modificaciones jurídicas que fortalecieron los depósitos de la población y creación de agencias regulatorias para vigilar al sector financiero (Peretz y Schroedel, 2009). La normativa regulatoria en Estados Unidos permitió la estabilidad del sistema económico y la regulación del capital financiero a nivel internacional.

De acuerdo con Sherman (2009) durante los 40 años posteriores de la Gran Depresión el marco constitucional tuvo pocos cambios, en cambio, los últimos 30 años, el sistema financiero internacional sufrió grandes modificaciones. A partir de 1970 el contexto mundial en materia de política económica cambió debido a los límites que mostró los planes económicos de corte keynesiano donde el Estado era el personaje vital de la dinámica económica. Los altos niveles de inflación, déficit fiscal y estancamiento económico fueron los síntomas del agotamiento del paradigma (Skildesky, 2018). La ofensiva contra la ineficiente intervención gubernamental condujo hacia el extremo. La nueva comprensión del mundo llevó al límite la idea sobre la eficacia del capital privado convirtiendo cualquier actividad púbica objeto de privatización. El consenso dominante en la economía estableció que la política monetaria podría llevarse a cabo sin apoyo de regulación financiera (Goodfiend y King, 1988).

Los golpes desde la conformación legislativa contra la regulación guiaron a la derogación de la Ley Glass-Steagall en 1999, episodio conocido como la Ley Gramm-Leach-Bliley. El gran pilar que daba soporte y seguridad a los depositantes fue eliminado bajo la premisa de modernización del sistema financiero. Este acto representó el mayor problema. La consolidación de instrumentos financieros sin regulación permitió la participación de diversos fondos de inversión magnificando sus operaciones gracias a la autonomía de la banca central cuyo papel fue importante en la relajación de la tasa de interés e incremento de la emisión monetaria. Ambos instrumentos motivaron el ascenso de las actividades financieras de alto riesgo.

En efecto, aquellos mecanismos que blindaron la actividad bancaria y financiera en favor de la población fueron removidos. El retiro de límites a la tasa de interés provocando que los créditos se dirigieran hacia la usura, el surgimiento de la banca múltiple posibilitando el uso de los depósitos de los contribuyentes en actividades especulativas, convertir los fondos para el retiro de los trabajadores en instrumentos financieros sujeto a las fluctuaciones de los mercados financieros y la creación de créditos sin respaldo bancario fueron las principales consecuencias de la desregulación financiera.

Dejar campo libre al crecimiento de actividades especulativas ha propiciado vulnerabilidad sistemática debido al surgimiento de burbujas, crecientes expectativos a futuro que se 
convierte en fracaso, pánico y finalmente crisis. Estos elementos gestaron la última gran crisis planetaria vinculada al sector hipotecario en 2008. Frente a la irresponsabilidad de las principales instituciones financieras, aseguradoras, bancarias y calificadoras surgió nuevamente la necesidad de regular, transparentar y disminuir el riesgo financiero: la Ley Dodd-Franken en 2010. Sin embargo, la administración de Donald Trump se encargó de su derogación en 2018 a fin de incrementar la competitividad de la economía estadunidense vía sector financiero al eliminar las restricciones a la creación del empleo y crecimiento económico. Esto es ejemplo de lo que sucede a nivel internacional; el ascenso y desregulación del sector financiero sobre el espacio real y el aparato político, son muestras de la financiarización.

\section{E1 proceso de financiarización}

La evolución del capitalismo ha desarrollado grandes transformaciones. Epstien (2005) indica que son 3 los cambios asociados: el neoliberalismo, la globalización y la financiarización. Del último fenómeno existen complicaciones para conceptualizarlo en una categoría única (Mader et al., 2019). Las múltiples definiciones son agrupadas por Ioannou y Wójcik (2019) en dos grandes conjuntos.

La primera surge a raíz de la definición más difundida que ofreció Epstien (2005) quien de manera general asocia el crecimiento de las actividades financieras sobre la estructura económica real a fenómenos como la creación deliberada del crédito hacia actividades de consumo y especulación, el papel de la banca en las sombras y el dinero creado de manera endógena por la banca privada sustituyendo a la banca central. El otro conjunto de conceptos se sustenta en otra definición que proliferó expuesta por Krippner (2005) quien identifica la financiarización como un nuevo patrón de acumulación en el que las ganancias se producen principalmente a través de canales financieros en lugar del comercio. Como resultado, el proceso implica una serie de transformaciones estructurales en la esfera política, económica y social.

En esta investigación, como se vislumbró en la introducción, se opta preferentemente por el segundo enfoque, aunque la compleja estructura de relaciones del sistema financiero requiere de las dos, por lo cual, ambas conceptualizaciones no son necesariamente excluyentes. La transformación del sistema (segunda definición) puede alimentar algún mecanismo de la primera definición.

En un breve recuento Baran y Sweezy (1966) vieron la financiarización como evolución histórica del capital, específicamente en el surgimiento del capital monopolista en los Estados Unidos a inicios del siglo XX. El exceso de recursos generados por las actividades industriales se tradujo en expansión del capital financiero. Mientras Hilferding (1973) apunta que la creación de estos monopolios y estructuras oligopólicas se debió a la articulación de las instituciones bancarias para financiar fusiones. Esta ruptura en la lógica del capital industrial provocó la división del capital en actividades financieras y productivas dejando de lado las inversiones de largo plazo en favor de las especulativas y de rentabilidad inmediata.

Otro estudioso de la dinámica del sector financiero fue Hyman Minsky (1992) quien en su obra argumenta el carácter endógeno de la inestabilidad del sector a causa del comportamiento empresarial por el afán de conseguir ganancias extraordinarias a partir de la expansión de actividades bursátiles. Por su parte Palley (2016) señala que este proceso se manifiesta en el 
incremento de la economía financiera por encima de la economía real produciendo transferencia de ingresos del sector real hacia las actividades especulativas y que finalmente contribuye a la concentración de ingresos e incremento de la desigualdad.

La financiarización es un concepto holístico y dinámico, por lo que sus impactos se manifiestan en el conjunto de sistemas sociales. Lapavitsas (2010) incorpora el papel de la banca privada en el otorgamiento de créditos a los hogares sin considerar las posibilidades de pago, mientras que Van der Zwan (2014) enfatiza en el cambio de las corporaciones hacia expectativas de ganancias futuras creando una cultura de "financiarización del cotidiano" gracias a la extensión del crédito en los hogares e incentivos para incorporar a las familias en la compra de activos financieros, principalmente en países con estructuras financieras desarrolladas.

Por otro lado, Jeannot (2020) señala que el ascenso del capital financiero tiene origen en una clase rentista, cuyo éxito reside en configurar un proceso de apropiación particular de rentas ${ }^{4}$ Como resultado, la clase rentista es causante de inestabilidad sistemática. En efecto, el sistema financiero se convierte en instrumento clave para el ejercicio del poder; los rentistas piensan el dinero como vehículo perfecto para realizar cualquier actividad especulativa en plena confianza de su inteligencia y habilidad para dominar el riesgo. Siguiendo al autor, el poder de esta clase llega a la esfera del gobierno al consolidar una estructura económica en favor de la captura de rentas. La financiarización de los servicios proveídos por el Estado es un rasgo esencial del fenómeno (Aalbers, 2019).

\section{La fragilidad y riesgos de la financiarización}

La acelerada integración financiera de los años noventa acompañada de la revolución de las tecnologías de la información y comunicación permitieron que los mercados internacionales se conectaran de manera automática. La visión favorable de la financiarización ha sido defendida desde distintas posturas: i) desde la tradición ortodoxa de la economía se piensa que los mercados en ausencia de cualquier institución del Estado llegan a resultados óptimos y eficientes. Es decir, los acuerdos entre individuos libres permiten alcanzar la mejor solución. La hipótesis de Fama ${ }^{5}$ (1991) sobre mercados eficientes ha sido el principal instrumento de validación; ii) bajo una visión armónica del mercado, la intermediación financiera se concibe como un vínculo entre ahorradores y prestamistas; iii) el crédito ofrecido es utilizado en actividades productivas; iv) existe un vínculo virtuoso entre el crecimiento financiero y desarrollo económico.

El listado de virtudes atribuidas a la financiarización es considerado por Assa (2020) como los mitos de las finanzas. La idea de eficiencia de los mercados financieros deviene de un marco analítico desarrollado en la economía ortodoxa donde no existe incertidumbre, no existe el Estado ni moneda, los agentes económicos tienen información completa y su comportamiento es racional $^{6}$, entonces, el precio de las acciones es el óptimo al reflejar toda la información del mercado y de las decisiones de los agentes. Esto desecha la posibilidad de

\footnotetext{
${ }^{4}$ A diferencia de las ganancias y salarios que proceden del proceso de acumulación, la renta deriva de la contraprestación en especie o monetaria sobre el usufructúo temporal de cierto bien. (Jeannot, 2020)

${ }^{5}$ De manera general señala que en condiciones de equilibrio los precios de los activos financieros reflejan de manera perfecta las condiciones del mercado.

${ }^{6}$ La economía neoclásica, la corriente dominante en la ciencia económica, parte de considerar el comportamiento de los individuos de manera racional. Es decir, los consumidores buscan en todo momento la satisfacción de su bienestar y los productores se ven motivados por maximizar las ganancias.
} 
crisis; en todo caso, la crisis es causa de factores exógenos. A través de este frágil esquema analítico se pugna por la ausencia de regulación y se justifican las crisis como resultado de sucesos que acontecen fuera de los mercados. El problema es el poder de estas figuras teóricas arcaicas para interpretar y defender el funcionamiento real de la economía financiera.

Referente al punto dos, la relación armónica entre ahorradores y prestamistas está entre dicho. Actualmente la cantidad de créditos que se otorgan no necesariamente tienen respaldo de garantía en los bancos, tan solo es resultado de un ejercicio contable (Nadal, y Aguayo, 2020). Actualmente la creación de dinero por parte de la banca privada sobrepasa de manera estratosférica la cantidad de depósitos. El resultado es la crisis del 2008 de la cual la economía mundial no logra salir. El mismo Tooze (2018) señala que la quiebra del banco de inversiones Lehman Brothers el 15 se septiembre del 2008 debió celebrarse como el día del libre mercado. Esto se vincula al punto tres, los créditos que provee la banca tienen mayores incentivos para canalizarse hacia el consumo suntuario o actividades especulativas ${ }^{7}$ a través de una compleja red de instrumentos e innovaciones financieras que ocultan a los compradores el riesgo del paquete adquirido. Por último, diversos trabajos señalan un vínculo entre el crecimiento del sector financiero y el desarrollo económico, sin embargo, las relaciones de causalidad se justifican principalmente en la evidencia empírica más no en la parte teórica (Yang, 2019 s Bucchi et al. 2019; Čižo et al, 2020)

La noción de un sistema financiero en armonía y promotor de las capacidades productivas se sostiene sobre pilares demasiados frágiles. En realidad, el ascenso de las finanzas sobre la estructura real de la sociedad ha dejado estragos severos convirtiéndose en un espacio de dominación, no sólo de los mercados, sino influyendo en las estructuras políticas dirigiendo la política económica en beneficio de las actividades rentistas y especulativas.

Al existir una economía numérica (Jeannot, 2020) y la mencionada economía ficticia el sistema económico y social corren serios peligros. La historia reciente nos demuestra que las consecuencias sociales han sido altísimas. Actualmente el obsesivo interés por el desempeño de los mercados financieros como el nivel de índices bursátiles, el incremento en los precios de los hidrocarburos en los mercados energéticos, el alza generalizada de los precios de las acciones gracias a un escenario menor incierto por la creación de la vacuna, acompañado de una política monetaria que provee dinero barato $^{8}$ al mundo y obsesionada por el control de la inflación ${ }^{9}$ se desvincula severamente de aspectos urgentes como desempleo, inversión, servicios públicos, etc. Además, acarrea otro tipo de riesgos como la intensificación de la

\footnotetext{
${ }^{7}$ Es importante señalar el papel de la política monetaria al facilitar las condiciones de especulación. En un entorno donde la tasa de interés de referencia de los bancos centrales es baja, la banca privada tiene condiciones ideales para solicitar préstamos. El exceso de liquidez incentiva a los jugadores financieros para canalizar los recursos hacia actividades especulativas en lugar de las productivas que requieren mayor tiempo de retorno. Para un ejemplo de este mecanismo véase el trabajo de Sherman (2009) y el papel de la Reserva Federal de Estados Unidos durante la crisis del 2008.

${ }^{8} \mathrm{~A}$ inicios del 2020 las reuniones de la Reserva Federal estadunidense apuntaban hacia el alza gradual de la tasa de interés. Sin embargo, la emergencia sanitaria provocó un retorno al esquema utilizado para reactivar la economía después del 2008 que fue la flexibilización monetaria. Es decir, inyección de miles de millones de dólares por parte de los bancos centrales hacia los mercados internacionales.

${ }^{9} \mathrm{Si}$ bien el control de la inflación es un elemento necesario en el desempeño económico se ha convertido en el único objetivo de la política monetaria en países latinoamericanos y varios países del mundo. Esto resulta de modelos económicos abstractos e irreales donde la estabilidad del sistema depende del control de precios. Habría que hacerse la pregunta planteada por Skildesky (2018) ¿realmente la estabilidad de una economía compleja del siglo XXI depende del control de precios? El mismo autor en el capítulo 13 de la obra referida demuestra lo anticuado de esta idea.
} 
financiarización en múltiples sectores de la economía y sobre la principal fuente de valor en cualquier sociedad: la naturaleza.

\section{Efectos de la financiarización sobre la naturaleza}

La financiarización de la naturaleza expresa una nueva forma de vulnerabilidad en países poseedores de inmensa biodiversidad (Isaacs y Kaltenbrunner, 2018). No obstante, los principales acuerdos internacionales en materia ambiental como el Acuerdo de París, los Objetivos del Desarrollo 2030, los programas de Naciones Unidas contra el Cambio Climático y para el Medio Ambiente sitúan en lugar estelar a las innovaciones tecnológicas e instrumentos de compensación ambiental como los mercados de carbono. Estos dos proyectos carecen de fundamentos sólidos si realmente intentan combatir el deterioro ambiental y evitar el incremento de la temperatura planetaria más allá de 2 grados centígrados. A continuación, una serie de argumentos en favor de la última afirmación.

En primer lugar, la noción de la tecnología se vincula a la posibilidad de transitar hacia un desarrollo sustentable. A saber, un esquema productivo que procure la creación de empleo, erradicación de la pobreza, inclusión social, bienestar, seguridad energética, solución de conflictos ambientales, competitividad económica y protección ecológica. La relación entre los diversos términos está sustentada mayoritariamente en el plano de las estadísticas que verazmente en la realidad ${ }^{10}$.

Las bondades de la tecnología sobre todo a una visión donde es posible remediar el daño ambiental gracias a los procesos de reciclaje y minimización del gasto energético. Sin embargo, no consideran los ciclos de la naturaleza y la irreversibilidad de las leyes naturales en términos energéticos; esto es la imposibilidad natural de restituir cualquier tipo de energía o materia, aspecto abordado de manera sobresaliente por Georgescu-Roegen (1971) ${ }^{11}$.

Otro elemento vinculado a la tecnología es la idea de reducir la huella ecológica siempre y cuando se consuman productos provenientes de procesos tecnológicos ecológicamente responsables. Sin embargo, esta idea genera sobre las personas un sentimiento de compensación (Sörqvist y Langeborg, 2019). Efectivamente, en lugar de reducir su consumo se ven motivados a incrementarlo en vista de que el daño por unidad de consumo es tan bajo que se justifica consumir más sin sentimiento de culpa. Este resultado es lo que se denomina la paradoja verde (Sinn, 2012) donde la innovación tecnológica incrementa la intensidad de explotación de la naturaleza.

A pesar de las dos objeciones presentadas, las grandes instituciones supranacionales apuestan al rol de la tecnología como la salvadora de los ecosistemas en el futuro. Nadal y Aguayo (2020) mencionan que la imposibilidad de esta idea radica en las condiciones actuales del capitalismo donde la inversión productiva y tecnológica no es atractiva prefiriendo canalizar los recursos monetarios en actividades rentistas. Si bien para la composición actual del

\footnotetext{
${ }^{10}$ Véase por ejemplo los trabajos de Redmond y Nasir (2020); Erdoğan et al. (2020); Zaidi et al. (2019) donde se vinculan las variables enlistadas cuya relación responde mayoritariamente al método de estimación confundiendo correlación y causalidad, en lugar de indagar de manera teórica-analítica en el vínculo de fenómenos correspondientes a la esfera social, ecológica y económica. ${ }^{11}$ Para el economista y matemático rumano todo proceso de producción tiene límites por las leyes naturales, en particular, el efecto de la entropía. Toda energía o materia utilizada en la producción ocasiona una pérdida energética irremediable sin importar el estado del progreso técnico o procesos de recuperación a través del reciclaje. La entropía incrementa mientras más intensivo en recursos naturales sea la producción, convirtiéndose en el elemento más dinámico de toda la producción. A diferencia de la teoría dominante en la economía donde no existen límites naturales, esta perspectiva replantea la teoría de la producción tradicional donde el cambio tecnológico es el aspecto vital para incrementar los rendimientos y combatir el deterioro ambiental. 
capitalismo no resulta prioritario invertir en tecnologías físicas, éstas se utilizan como referentes para crear una serie de activos financieros idóneos para la especulación, velo que esconde los intereses de rentistas bajo la idea de sustentabilidad ambiental. A este punto se regresa más adelante.

Otro elemento clave en los planes de acción internacional es la creación de mercados de carbono. Este instrumento expreso de la financiarización (Assa, 2020) en idea permite a cada país tener un límite de emisiones anuales de dióxido de carbono. En caso de superarlo, podrá negociar con otros países quienes no lo hayan utilizado en totalidad. En términos estadísticos, el exceso de un país se compensa con el faltante de otra nación, logrando el equilibrio numérico. La idea detrás de este mecanismo es la mercantilización de la naturaleza.

En efecto, reducir la complejidad biológica a términos estrictamente económicos permite introducir a la naturaleza al espectro del resto de mercancías sujetas a la oferta y demanda de mercado. A su vez, la expresión monetaria permite configurar una serie de instrumentos financieros a su alrededor haciendo participes a los países del mundo en un mercado común integrado para intercambiar saldos de emisiones contaminantes. Por lo cual, retorna la idea de compensación a través de su equivalente monetaria. En consecuencia, la naturaleza como principal creador de valores culturales y sociales (Valdés, 2004) se limita a la expresión cuantitativa, además de beneficiar a los intermediarios financieros que facilitan esta operación.

La acelerada integración financiera desarrolló un conjunto de complejos activos en el corto plazo para lograr comercializar acciones relacionadas con bienes naturales y no sólo un mercado financiero de bonos de carbono de escala mundial como se presentó en líneas arriba. Dentro de la diversa gama de innovaciones financiera el mecanismo más utilizado es la creación de mercados a futuros (Meydroidt, 2018; Keucheyan, 2018). El grado de penetración de la financiarización se manifiesta en diversas escalas. El caso más estudiado se vincula a medios naturales que forman parte de la canasta básica alimenticia y energética de poblaciones en el mundo, los denominados commodities.

A partir del año 2000, de acuerdo con Falkowsky (2011) se vivió una escalada en los precios de los productos como el maíz, trigo, café, azúcar, carne porcina, oro, petróleo, gas, oro, entre otros, además del precio de las viviendas. Las variaciones de precios se justifican principalmente por la creciente demanda de países asiáticos e industrializados, aun cuando existe evidencia para señalar que se debe a la influencia de manipulación de acaparadores, grandes comercializadoras y especulación financiera ${ }^{12}$.

Los mercados a futuro son un motor clave en la degradación medioambiental. El régimen de acumulación basado en las rentas de instrumentos bursátiles con respaldo en commodities acelera la destrucción de la naturaleza. El poder de los contratos a futuros permite hipotecar grandes volúmenes de producción durante un lapso de tiempo y crear una gran cantidad de activos financieros a partir de una expectativa de producción.

Los activos financieros garantizan cierto nivel de producción con el fin de obtener rentas favorables. El endeudamiento compromete los rendimientos de la naturaleza durante varios años en vista de garantizar precios a favor del inversor, en contraparte, la intensidad de explotación incrementa. La búsqueda de rentabilidad genera mayores niveles de extracción comprometiendo la recuperación de los ciclos naturales. Mantener la rentabilidad de la tierra

\footnotetext{
$12 \mathrm{Al}$ respeto véase Cheng y Xiong (2014).
} 
implica incrementar el uso de pesticidas, químicos o mutaciones genéticas además de proliferación de monocultivos que terminan con las propiedades naturales de la tierra y contra la diversidad de especies. Bajo la idea de soberanía alimentaria se ha defendido este proceder invisibilizando múltiples prácticas desleales.

El poder de grandes comercializadora para acceder a financiamientos, además en un entorno como el actual, como se mencionó, con dinero a bajo costo en el sistema de pagos internacional, les permite influir sobre los precios nacionales e internacionales a través de la especulación. Al respecto, Randall Wray (2008) señala que el ascenso de los precios de las materias primas en los últimos años se debe a prácticas de acaparamiento. El auge de la burbuja incentiva inversiones en estos instrumentos y compromete mayores niveles de producción a futuro. Esta renta producto de la intermediación queda en manos de los inversores institucionales. En cambio, la caída de los precios acomete contra los productores quienes esperaban vender a un precio superior y que, debido a los movimientos de precios, por factores financieros, incurren en pérdidas.

Los daños de la financiarización en bienes y energías básicas implican mayores niveles de estrés ecológico y daños en el patrimonio de productores, en vista de un panorama vulnerable, quedan sometidos al poder de las grandes comercializadoras. Además, en las etapas de auge, la codicia por privatizar las tierras bajo el control de las comunidades incrementa generando conflictos por la defensa de la propiedad (Meyfroidt, 2018)

Otro mecanismo donde opera la financiarización es la inversión en tecnologías renovables. Como se mencionó algunas líneas anteriores, el consenso a nivel internacional para combatir el cambio climático y arribar a un esquema sustentable es el fomento del desarrollo técnico. El principal problema con esta visión es la ausencia del progreso financiero en la exploración de nuevas fuentes energéticas.

Efectivamente, la explotación de materiales raros es clave para la revolución digital. Balaram (2019) enlista los 17 elementos químicos presentes en dispositivos tecnológicos, computadoras, baterías para autos eléctricos, iluminación, resonancia magnética y otros dispositivos vinculados a procesos de alta tecnología y elementos de la transición energética, cuyo funcionamiento depende principalmente de minerales obtenidos a través la explotación de minas. Dawid (2019) señalan diversos conflictos en América Latina por la disputa del litio en países como Bolivia, Argentina y Chile, mientras Carpintero y Naredo (2018) apuntan el surgimiento del neoextractivismo liderado a nivel mundial por China en busca de componentes estratégicos para la industria informática y tecnologías renovables.

Bajo el argumento de las tecnologías renovables y productos energéticamente sostenibles puede intensificarse el conflicto social e incrementar el desastre ambiental a nivel planetario, asimismo incentivar la participación de inversores financieros en actividades relacionadas a la extracción de minerales raros y promoción de tecnologías renovables que siguen siendo altamente contaminantes. Siguiendo a Martin e Iles (2020) la competencia por los minerales raros encargadas del tránsito energético ha dejado a su paso conflictos por la tierra, destrucción del paisaje y explotación abusiva de otros elementos naturales como el agua.

Otro mecanismo en que opera la financiarización es deslindar a las empresas de sus responsabilidades ecológicas. De acuerdo al Carbon Majors Report (2017) cien empresas son generadoras del 70\% de las emisiones de dióxido de carbono en el mundo principalmente relacionadas al sector de hidrocarburos. A pesar de lo contundente de las cifras, cierto 
segmento de empresas se dedica a encontrar ganancias de la catástrofe ecológica a través de la creación de estructuras de seguros y coberturas contra desastres ambientales para las empresas y familias. Vale decir, por un lado, empresas que producen emisiones de efecto invernadero nunca antes vistas en la historia de la humanidad (Balzani, 2019) y por otro, empresas del sector financiero creando un mercado contra riesgos.

Keucheyan (2018) apunta en últimas fechas al desarrollo de instrumentos que blindan de daños por cualquier catástrofe natural. Esto a su vez incentiva a las empresas por incluir en sus activos financieros las coberturas de seguros para seguir operando y cumplir con las normas ambientales. Esta secuencia no termina allí, Höck et al. (2020) mencionan que actualmente las compañías han incorporado en sus balances la noción de contabilidad ambiental. Esto es, una herramienta donde las empresas reportan, a nivel contable, esquemas de reducción de impacto ecológico con el fin de atraer a consumidores con cierto nivel de conciencia del problema ambiental e igualmente ofrecen seguros a las familias contra cualquier tempestad. Estos nuevos espacios acaparan la atención de diversas instituciones crediticias y promueven la innovación financiera a cambio de captar dinero con alto riesgo de crear burbujas especulativas. La razón se debe a la posibilidad casi nula de acertar en el pronóstico sobre cierto acontecimiento natural.

El uso de seguros y coberturas debería ser altamente cuestionado por la visión detrás del mecanismo en que operan. Esta perspectiva solapa el agresivo modo de producción de las empresas porque mientras mayor contaminación exista las posibilidades de generar ganancias incrementa. Dicho proceder lucra con el acelerado nivel de daño ecológico creando una atmosfera comunicativa donde justifican su pertinencia al generar instrumentos mitigadores del cambio climático.

No obstante, la misma dinámica de acción los lleva a una gran contradicción. En la práctica, a medida que incrementa el peligro ambiental, el sector financiero se ve motivado a incrementar el número de instrumentos e innovaciones financieras para diversificar el riesgo y ampliar la cobertura de clientes, tanto empresas y familias. A su vez, al enfrentarse a fenómenos extremadamente aleatorios, la especulación de los instrumentos incrementa. El ascenso de la estructura financiera y la velocidad de deterioro ecológico crecen sincrónicamente y seguiría el mismo camino que el sector inmobiliario durante el 2008. En otros términos, una estructura inmensa que supera la posibilidad real de la economía para sostenerlo.

El problema ambiental llegará al punto donde las exigencias de pago de las diversas pólizas y seguros será mayor a la capacidad financiera de solventarla; nuevamente vendría un colapso donde miles de familias perderán su patrimonio. De vital importancia la preocupación de los gobiernos frente a esta manifestación de la financiarización. Existe un riesgo a causa de la creciente estructura especulativa en este sector que, como afirmó Stglitz (2015) en lugar de gestionar el riesgo, el riesgo se creó. En caso de no poner límite a este régimen de acumulación basado en la captura de rentas y mientras el daño ambiental no se detenga, el camino por delante es demasiado sombrío.

Las instituciones financieras observan en los campesinos grandes clientes para sus instituciones bajo el discurso de inclusión financiera. Frente al entorno de volatilidad en los precios en las mercancías agrícolas básicas generado por el poder de control de las comercializadoras en el mercado internacional, los campesinos se ven obligados a adquirir 
coberturas de protección contratando bajo condiciones desfavorables. Por otro lado, si las comercializadoras emiten un mayor volumen de compromisos financieros en los mercados a futuro, los pequeños productores requerirán de financiamiento para incrementar el volumen de producción, creando mayores presiones al medio ambiente. La vulnerabilidad y destrucción es un rasgo esencial de la proliferación de actividades especulativas en áreas que buscan la explotación de la naturaleza.

\section{El peligro de la financiarización frente a la pandemia}

Durante los últimos años se han concentrado las preocupaciones del mundo en dos grandes problemáticas: el estancamiento económico y la crisis medioambiental. Frente a los retos que implica contrarrestar ambos fenómenos, se ha planteado construir un conjunto de políticas que evocan al paquete de reformas instauradas durante los años treinta en Estados Unidos para superar la gran depresión. En busca de una versión ambiental del New Deal la senadora Alexandria Ocasio-Cortez nombró el Green New Deal.

El nuevo pacto medioambiental constituye una hoja de acción que insta a los gobiernos a construir un plan de transición energética mientras se lucha por la desigualdad económica. Los principales mecanismos pueden resumirse en: i) disminución de las emisiones de dióxido de carbono em 100\% para 2050; ii) modernización tecnológica a nivel sectorial que promueva el uso de energías renovables; iii) incentivos fiscales para promover el cambio tecnológico con miras a la mitigación y adaptación frente al cambio climático; iv) proveeduría de servicios energéticos a los hogares de fuentes renovables; v) creación de plazas de trabajo con alta remuneración resultado del impacto tecnológico del tránsito energético; vi) flexibilización para la creación de mercados de carbono e implementación de impuestos a emisiones con la finalidad de transferir recursos monetarios a las poblaciones afectadas; vii) creación de fideicomisos para financiar las diversas acciones contra el cambio climático.

Esta serie de medidas son ampliamente promovidas como el camino que las economías del mundo deberían seguir. Como se observa, el plan de acción internacional sigue la misma línea argumentativa y complementaria al resto de acuerdos internacionales vigentes. Como se ha intentado mostrar, los pilares que sustentan los mecanismos carecen de comprensión referente a elementos vitales como el impacto de las leyes naturales sobre los procesos sociales y la homogenización de múltiples esferas de acción a partir del criterio monetario.

Ahora bien, un elemento persistente y protagónico es el impulso hacia del cambio técnico como figura protagónica para mitigar el cambio climático, crear empleos, reducir la desigualdad e incrementar el crecimiento económico. Sin embargo, como se ha pretendido argumentar, la integración de los mercados y gobiernos se han convertido en espacios ideales para los rentistas. La aceleración de la financiarización consolida un esquema de acumulación y configura un esquema de desarrollo destructivo y depredador contra el medio ambiente. Igualmente, la idea de desarrollo imperante justifica la integración de economías emergentes en el esquema rentista con la finalidad de conseguir recursos económicos para llevar a cabo sus objetivos en materia ambiental a costa de intensificar y comprometer ciertos volúmenes de minerales, energético y materias primas hipotecando el futuro de la naturaleza.

El mundo transita ciegamente hacia pactos medioambientales que suponen integrar el desarrollo económico y el cuidado ecológico. El término de sustentabilidad se ha convertido en una categoría de uso común cuyas implicaciones en la realidad son débiles. El esquema 
tradicional no cuestiona el actual estilo de producción incluso acepta el dominio de la estructura económica por parte de las finanzas.

El discurso de la sustentabilidad imperante opera bajo el esquema de racionalidad ambiental donde los individuos delegan la solución a los mecanismos de mercado (Leff, 2004). Muchas veces se acusa la resistencia "al progreso y desarrollo" de ciertos comunidades originarias o pequeños productores agrícolas a las bondades que brinda la financiarización, aun cuando estas actividades resultan en dominio de los intereses rentistas sobre la naturaleza y la vida de las personas.

La situación que afronta la humanidad por la pandemia a raíz del virus SARS-CoV-2 necesitará de respuestas en materia económica a corto plazo. Frente a la coyuntura es probable que se piense en rutas de emergencia como el Green New Deal debido a lo atrayente de su discursiva tanto en el aspecto político y ámbito social, generando entusiasmo dentro de un ánimo de social deprimido.

Aun cuando resulte una luz alcanzable sus impactos profundizarían el proceso de financiarización contra la naturaleza. En efecto, seguir ciegamente los principios del nuevo pacto verde alimentaría el papel del sector financiero en las múltiples escalas que se mencionaron en el apartado anterior. Además, la fragilidad de los Estados nacionales después políticas neoliberales durante los últimos años (Skildesky, 2018) requerirá respaldo de los mercados financieros internacionales quienes, bajo el discurso de impulso al desarrollo expresado en empleos, crecimiento y sustentabilidad ambiental, incrementarán su dominio sobre la estructura real además de los riesgos sociales y ambientales. Es relevante reflexionar sobre las rutas que podría tomar los gobiernos del mundo para la era post-covid. Algunos puntos iniciales de diálogo que podrían ser parte de un esquema fuerte de sustentabilidad frente a la financiarización y la inherente capacidad del capitalismo de aniquilar el ambiente se desarrollan en el apartado final.

\section{Conclusiones}

El actual esquema de sustentabilidad profundiza e incentiva el desarrollo de actividades intensivas en el uso de la naturaleza gracias a que cualquier daño ecológico es posible remediarlo a través del dinero. Este trabajo intenta demostrar los peligros de la financiarización de la naturaleza, en particular, el riesgo que existe de que se convierta en estrategia internacional para salir del estancamiento generado por la pandemia como la que promueve el Green New Deal.

Todo riesgo incrementa debido a que tanto los empresarios, individuos y gobiernos deslindan sus responsabilidades ecológicas en tanto sigan fielmente los diversos mecanismos prevalecientes en los acuerdos internacionales sin ser conscientes de que la crisis ecológica en términos de materia y energía se profundiza, aunado a la creciente estructura rentista especulativa que resulta beneficiada del incentivo de rentabilidad. El entorno macroeconómico promovido por los bancos centrales que se ha encargado de llenar los mercados de dinero a bajo costo a través de la flexibilización monetaria es un espacio ideal para estas actividades y que seguirá siendo clave en los meses venideros como estrategia de recuperación posterior a la pandemia.

La contingencia sanitaria, dentro de sus múltiples factores, es resultado del dominio de los seres humanos sobre la naturaleza. La explotación de diversas especies, el comercio ilegal, 
modificación genética entre otras consecuencias son causa de un factor clave: la consideración de la naturaleza en su expresión puramente mercantil y monetaria.

El proceso de financiarización prioriza la creación de valores financieros sobre las mercancías, por lo cual, los principios que podrían fungir como alternativa parten de reconsiderar esta visión. En primer lugar, cambiar radicalmente la consideración de la naturaleza en términos monetarios. Existe un gran debate sobre la importancia de valorar la biodiversidad como creadora de valores culturales y sociales ${ }^{13}$.

Debe concebirse la relación ser humano-naturaleza como miembros de un sistema superior donde cualquier sistema social es parte del sistema ecológico, no para servirse de ella sino comprender que la sobrevivencia de cualquier sistema cultural y de la vida misma depende de la naturaleza. A nivel social permitiría al ciudadano crear una idea fuerte sobre el cuidado ambiental y no pensar que el reciclaje es una verdadera estrategia ecológica.

Otro elemento es la consideración y construcción de diversas formas alternativas de desarrollo partiendo de la pregunta ¿Para quién? Concebir otras formas de desarrollo depende de la diversidad cultural y múltiples ópticas existentes. En particular, el respeto hacia esquema de desarrollo implementadas por comunidades rurales en zonas del mundo como América Latina han demostrado crear estructuras mercantiles fuera de la racionalidad ambiental en relación con el capitalismo dentro de un entorno de respeto ecológico. Estas ideas no son discursos morales sino constituyen diálogos reales que permitirían transitar a una situación favorable.

Por último, a pesar de respuestas a nivel particular, se requiere prácticas al nivel donde existen los grandes intereses económicos y políticos, sin embargo, no están desarticulados. El cambio de consideración de la naturaleza fuera de criterios mercantiles retira el incentivo a los rentistas de apreciar los instrumentos financieros como valor monetario. El papel de los Estados tendría que ser el desaliento a las actividades especulativas. Para ello se requiere configurar una estructura económica orientada a las ganancias de productividad (Jeannot, 2020) y colocar restricciones a nivel mundial de los capitales especulativos. La promoción de la recuperación económica post COVID-19 tendría que orientar sus esfuerzos hacia la creación de trabajo y reducir la desigualdad a partir de criterios diferentes y no utilizar el argumento ecológico de por medio.

Como se mencionó en el apartado correspondiente, si no se toma el problema real de la financiarización del medio ambiente traería problemas graves, principalmente en un futuro donde la ausencia de regulaciones provocaría un daño mayor y las catástrofes serían frecuentes. El papel del Estado en la regulación, desincentivo de la especulación y mecanismos para retirar el valor monetario de la naturaleza tendrían que ser estrategias para un futuro que intentará dejar en recuerdo la pandemia en un contexto de globalización del siglo XXI.

\section{Referencias}

Aalbers, M. (2016). "Financialization”. En D. Richardson, N. Castree, M. Goodchild, A. Kobayashi and R. Marston (eds.) The International encyclopedia of geography: People, the earth, environment and technology. Oxford: Wiley.

Assa, J. (2020). "Liquidating Society and the Planet: Financialization vs. Sustainability". Disponible en https://www.researchgate.net/profile/Jacob_Assa2/publication/344191278_Liquidating_Society_and

${ }^{13}$ Véase el trabajo clásico de Leopold (1949) 
_the_Planet_Financialization_vs_Sustainability/links/5f5a285192851c078958bfa5/Liquidating-

Society-and-the-Planet-Financialization-vs-Sustainability.pdf.

Balaram, V. (2019). "Rare earth elements: A review of applications, occurrence, exploration, analysis, recycling, and environmental impact". Geoscience Frontiers, 10(4): 1285-1303.

Balzani, V. (2019). "An energy transition to save the planet". Substantia, 3 (2): 1-10.

Banco Mundial (2020). Global Economic Prospects June 2020. Disponible en https://www.worldbank.org/en/publication/global-economic-

prospects\#: :text $=$ Global $\% 20$ Outlook,-

COVID $\% 2$ D $19 \% 20$ has\&text $=$ The $\% 20$ baseline $\% 20$ forecast $\% 20$ envisions $\% 20$ a,economies $\% 20$ will $\% 2$ 0shrink \%20this\%20year

Baran, P. y Sweezy, P. (1966). El capital monopolista: un ensayo sobre la economía estadounidense y el orden social. Madrid, Siglo XXI editores.

Bruckmann, M. (2016). "La financierización de la naturaleza y sus consecuencias geopolíticas". América Latina en Movimiento, 517: 13-16.

Bucci, A., La Torre, D., Liuzzi, D. y Marsiglio, S. (2019)." Financial contagion and economic development: An epidemiological approach". Journal of Economic Behavior and Organization, 162: 211-228.

Carbon Majors Report (2017). The Carbon Majors Database. Disponible en https://6fefcbb86e61af1b2fc4c70d8ead6ced550b4d987d7c03fcdd1d.ssl.cf3.rackcdn.com/cms/reports/documents/000/002/327/ori ginal/Carbon-Majors-Report-2017.pdf?1501833772

Carpintero, O., y Naredo, J. M. (2018). "Sobre financiarización y neoextractivismo". Papeles de relaciones ecosociales y cambio global, 143: 97-108.

Chang, H. (2012). 23 things they don't tell you about capitalism. Bloomsbury Publishing

Cheng, I. y Xiong, W. (2014). "Financialization of commodity markets". Annual Review Financial Economics, 6(1): 419-441.

Čižo, E., Lavrinenko, O., y Ignatjeva, S. (2020). "Analysis of the relationship between financial development and economic growth in the EU countries". Insights into Regional Development, 2(3): 645-660.

Dawid, D. (2019). Naturaleza y Conflicto: La explotación de recursos en América Latina. Ediciones Akal.

Epstein, G. (Ed.). (2005). Financialization and the world economy. Edward Elgar Publishing.

Erdoğan, S., Yıldırım, D. y Gedikli, A. (2020). "Natural resource abundance, financial development and economic growth: An investigation on Next-11 countries". Resources Policy, 65: 101-159.

Falkowski, M. (2011). "Financialization of commodities". Contemporary Economics, 5(4): 4-17.

Fama, E. (1991). "Efficient capital markets: II". The journal of finance, 46(5): 1575-1617.

Georgescu-Roegen, N. (1971). The entropy law and the economic process. Cambridge Press.

Goodfriend, M. y King, R. (1988). Financial deregulation, monetary policy, and central banking. Federal Reserve Bank of Richmond Working Paper.

Hilferding, R. (1973). El capital financiero. Tecnos.

Höck, A., Klein, C., Landau, A. y Zwergel, B. (2020). "The effect of environmental sustainability on credit risk". Journal of Asset Management, 21:1-9.

Ioannou, S., y Wójcik, D. (2019). “On financialization and its future. Environment and Planning”. Economy and Space, 51(1): 263-271.

Isaacs, G., y Kaltenbrunner, A. (2018). "Financialization and liberalization: South Africa's new forms of external vulnerability". Competition and Change, 22(4): 437-463.

Jeannot, F. (2020). "La competitividad imperfecta en el umbral del coronavirus". Contribuciones a la Economía, Abril 2020: 1-52

Keucheyan, R. (2018). "Insuring climate change: new risks and the financialization of nature". Development and Change, 49(2): 484-501.

Kindleberger, C. y Aliber, R. Z. (2005). Manias, panics and crashes: A bistory of financial crisis. Palgrave Macmillan.

Krippner, G. (2005). "The financialization of the American economy". Socio-economic Review, 3(2): 173-208.

Lapavitsas, C. (2010). Financialisation and capitalist accumulation: structural accounts of the crisis of 2007-2009. Research on Money and Finance Discussion Papers, (16).

Leff, E. (2004). Racionalidad ambiental: la reapropiación social de la naturaleza. México: Siglo XXI.

Leopold, A. (1949). A Sand County almanac, and sketches here and there. Oxford University Press.

Mader, P., Mertens, D., y Van der Zwan, N. (2019). Financialization: An Introduction. The Routledge international handbook of financialization. Abingdon: Routledge. 
62 Los efectos de la financiarizacion sobre la naturaleza en el contexto de la pandemia COVID-19

Martin, A. y Iles, A. (2020). "The Ethics of Rare Earth Elements Over Time and Space”. International Journal for Philosophy of Chemistry, 26: 5-30.

Meyfroidt, P. (2018). "Financialization and the Forestry Sector". En: C. Farcy, E. Rojas-Briales e I. de Arano (Eds.). Forestry in the Midst of Global Changes. CRC Press.

Minsky, H. (1992). The financial instability bypothesis. The Jerome Levy Economics Institute Working Paper, (74).

Nadal, A., y Aguayo, F. (2020). Los motores de la degradación ambiental: el modelo macroeconómico y la explotación de los recursos naturales en América Latina. Ciudad de México: CEPAl.

Palley, T. (2016). Financialization: the economics of finance capital domination. Springer.

Peretz, P. y Schroedel, J. (2009). "Financial regulation in the United States: Lessons from history". Public Administration Review, 69(4): 603-612.

Redmond, T. y Nasir, M. (2020). "Role of natural resource abundance, international trade and financial development in the economic development of selected countries". Resources Policy, 66: 10-15

Sherman, M. (2009). A short history of financial deregulation in the United States. Center for Economic and Policy Research, (7).

Sinn, H. (2012). The green paradox: a supply-side approach to global warming. MIT Press.

Skidelsky, R. (2018). Money and Government: A Challenge to Mainstream Economics. United Kingdom: Penguin.

Sörqvist, P. y Langeborg, L. (2019). "Why people harm the environment although they try to treat it well: An evolutionary-cognitive perspective on climate compensation". Frontiers in psychology, 10: 1-5.

Stiglitz, J. (2015). La gran brecha: qué hacer con las sociedades desiguales. Taurus.

Tooze, A. (2018). Crashed: How a decade of financial crises changed the world. Penguin.

Valdés, M. (2004). Naturaleza y valor. Fondo de Cultura Económica.

Van der Zwan, N. (2014). "Making sense of financialization". Socio-Economic Review, 12(1): 99-129.

Wray, L. (2008). The commodities market bubble: money manager capitalism and the financialization of commodities. Public Policy Brief, (96).

Yang, F. (2019). "The impact of financial development on economic growth in middle-income countries". Journal of International Financial Markets, Institutions and Money, 59: 74-89.

Zaidi, S., Wei, Z., Gedikli, A., Zafar, M., Hou, F. e Iftikhar, Y. (2019). “The impact of globalization, natural resources abundance, and human capital on financial development: Evidence from thirty-one OECD countries". Resources Policy, 64: 14-46. 